\title{
NOTAS SOBRE LA HISTORIA ECONÓMICA DE LA CONSOLIDADA, S.A. (1936-1991)
}

\author{
Por \\ Salvador Corrales C.*
}

\begin{abstract}
RESUMEN
La Consolidada, S.A. fue una empresa pionera del ramo de la industria siderúrgica nacional, constituida a principios de siglo en la ciudad de México por iniciativa privada americana, que vio en nuestro país un mercado para la producción y comercialización de productos de acero. Una vez que concluyen los movimientos armados de la revolución, se recupera el daño de la crisis de 1929 y se define el futuro del México posrevolucionario, esta empresa contruye una planta de fundición en la ciudad de Piedras Negras, Coahuila para responder a sus necesidades de expansión y atender las demandas del mercado. Desde 1936 se hacen los primeros trabajos para la construcción de dicha planta, y su primera colada la obtiene en mayo de 1938. A pesar de su tamaño, la principal forma de integración a la economía de la ciudad fue a través de los salarios pagados a sus trabajadores. Aun cuando no se ha investigado toda su historia económica, objeto del presente ensayo, la información disponible hasta hoy demuestra que no hay una gran empresa en esta ciudad, mucho menos un grupo de empresarios prosperos, cuya fortuna pueda atribuirse a la empresa acerera cerrada en 1991 como resultado del proceso de reestructuración industrial.
\end{abstract}

\begin{abstract}
La Consolidada, S.A. was a pioneer organization in the national iron and steel industry, set up in the early century in Mexico City by American private initiative that considered our country a market for production and commercialization of steel products. Once the revolutionary movement ended, the economical crisis of 1929 was overcome, and the future of Mexico after the Revolution is defined, this organization builds a smelting plant in the city of Piedras Negras, Coahuila, to respond to their necessities of expansion, and to attend marker's demand. In 1936 begins the construction of the plant, and its first washing was accomplished in May 1938. Despite of its size, the main sourer of integration into the economy of the city was through the salaries paid to its employees. Although its economic history has not been investigated in the whole objective of this work, the available information up until now shows that there is not a big organization in this city, and neither a group of prosperous bisiness men, whose fortune can be attributed to the steel industry closed down in 1991 as a result of the industrial restructuration process.
\end{abstract}

Investigador de El Colegio de la Frontera Norte en Piedras Negras, Coahuila.

N. del E. Este artículo se aprobó en junio de 1997 para ser publicado en Estudios Fronterizos. 


\section{BOSQUEJO}

Una vez concluidos los efectos de la gran depresión de 1929, en medio de los conflictos de lo que sería el México posrevolucionario de cara al resto del mundo, se iniciaron los primeros trabajos para la instalación de una fundición que posteriormente se convertiría en una de las principales empresas siderúrgicas mexicanas que apoyaron con materias primas, insumos y herramientas a nuestra industrialización en su etapa inicial.

La Consolidada, S.A. colocó la primera piedra de su sucursal en la ciudad de Piedras Negras, Coahuila en 1936 y la primera colada ${ }^{1}$ la obtuvo el 28 de mayo de 1938 (Reyes, 1996). Con esta planta abastecería de lingotes de acero a su empresa matriz ubicada por la calzada La Ronda, en la colonia Peralvillo de la Ciudad de México, cuya presencia en el mercado mexicano había experimentado ya 31 años elaborando productos de acero para un mercado virgen, cada vez con mayores demandas de toda la gama de productos que necesitaba en aquellos años nuestra incipiente industria metal-mecánica y de la construcción.

El estadounidense Harry Wright vino a México a principios de sìglo

[...] con la idea de instalar una fundición que aprovechara la chatarra de los ferrocarriles y de las minas, se asoció con Charles Seifert, que a su vez era dueño de un establecimiento de manufacturas de hierro llamado "Valley Metals", para organizar una fundición que denominaron La Consolidada, S.A., con un capital inicial de tres millones de pesos (Zapata y Toledo, 1987).

En un principio la instalaron en Nonoalco, Distrito Federal y su primer nombre fue Consolidated Iron \& Equipment Company; en el año de 1913 cambió de nombre a Consolidated Rolling Mills \& Foundries Company; se castellaniza con el nombre de La Consolidada, S.A. en 1918.

$\mathrm{La}$ instalación de esta planta fundidora de acero (la segunda en su género después de Fundidora Monterrey) para aprovechar el fuerte auge de la industria metal-mecánica, de la construcción y manufacturera en general, cuya demanda de acero tenía que abastecerse con importaciones de Estados Unidos, propicio una controversia muy importante que podría decirse, motivó el cambio de estrategia de crecimiento económico. La industria manufacturera mexicana dependía tanto del exterior que "[...] entre 1910 y 1920 las importaciones de hierro y acero se [incrementaron]

1 Se entiende por colada al proceso de extracción de arrabio del al to horno para depositarlo en los hornos de aceración. De igual modo, de los hornos de aceración se extrae el acero y se vacía a los moldes lingoteros o a las ollas del sistema de colada continua. 
en un 305\%" (Zapata y Toledo, 1987). A la incipiente industria siderúrgica mexicana, encabezada por Fundidora Monterrey, S.A., la apertura de las fronteras a las importaciones de esta industria le ponía obstáculos a sus planes de expansión, y con mayor razón a la pequeña empresa La Consolidada, S,A., construida con un capital más modesto.

Varios fueron los intentos de Harry Wright para convencer a los presidentes Ålvaro Obregón y Plutarco Elías Calles para que incrementaran los aranceles a las importaciones de estos productos. La fortaleza del sector minero, el más poderoso en aquellos años y principal importador de maquinaria, refacciones y productos de acero en general, opuso resistencia, haciendo fracasar a Harry Wright, quien alguna vez le dijo a Obregón que:

"La industria del acero es básica para el desarrollo industrial de cualquier país [...] de tal manera que si un país cuenta dentro de sus fronteras con una industria siderúrgica desarrollada, se independizará del exterior y logrará una verdadera soberanía industrial" (Zapata y Toledo, 1987).

Años después, y como parte de la nueva estrategia de crecimiento económico propiciado por la gran crisis de 1929 y la segunda guerra mundial, que motivó una mayor participación del Estado en la economía, el proteccionismo se consolidó y con él las condiciones para que la industria nacional se desarrollara, constituyendo una época de bonanza, acumulación de capital y constitución de grandes empresas paraestatales y privadas. La lucha de La Consolidada, S.A. por modificar las fronteras a las importaciones tuvo mucho peso en este cambio que llevó al incremento de los aranceles y, posteriormente, a la aplicación de permisos de importación.

Entre los criterios para la ubicacion de la planta fundidora en Piedras Negras, Coahuila se consideró la cercanía a la materia prima que serviría para fundir; en esos años existía abundante chatarra proveniente de el sur de Estados Unidos y Piedras Negras era el lugar más práctico por la presencia del ferrocarril, que se utilizaría para transportar tanto la materia prima como el producto terminado. Cuenta Roberto Reyes ${ }^{2}$ en sus Recuerdos que Nuevo Laredo, Tamaulipas estuvo a punto de ser la sede de esta empresa, pero sus autoridades realizaron las gestiones muy tardiamente ya

2 Obrero sin preparación técnica al guna que ingresó a la compañía en 1936, cuando se hacian los trabajos de construcción de la planta. Su lúcida inteligencia y su amor al trabajo y a la empresa lo llevaron a ocupar el puesto de superíntendente desde 1939 hasta 1973 , año en que se jubiló. A él le debemos parte importante de esta historia. 
que para entonces se habían realizado grandes inversiones en dólares en las instalaciones cuando ofrecieron a Harry Wright todos los servicios y facilidades: exención de impuestos por varios años y terrenos apropiados para construirla.

La Consolidada, S.A., mejor conocida en la comunidad como "La Concha", vino a llenar el vacío que dejo La Maestranza, taller del ferrocarril que hacía reparaciones a los vagones. La Maestranza era un gran taller con muchos trabajadores, hábiles tomeros que llegaron a construir cañones para el servicio de la revolución (Sánchez, 1990). Cuentan que esta empresa fue cerrada por Plutarco Elías Calles como castigo al pueblo de Piedras Negras, que no acudió a recibirlo cuando realizaba su campaña para la presidencia de la República; así lo atestiguan los pocos sobrevivientes de la generación de los años veinte. Indignado, Calles les aseguró que tomaría represalias; las causas reales del cierre de La Maestranza todavía se desconocen.

Con el cierre de La Maestranza, la ciudad experimentó un vacío económico; una carencia de fuentes de empleo que por fortuna, La Consolidada, S.A. vendría a redimir y a capitalizar la experiencia laboral que sus empleados ya habían acumulado en el manejo de máquinas, como fueron: Fructuoso Gonzalez ("Tocho"), Rosendo Veloz, Gabriel Bustamante, Nicolas O'Brian, Alfredo Gómez y Francisco Patiño (Reyes, 1996); todos ellos fueron contratados por la nueva empresa desde que inició las instalaciones. La Consolidada. S.A. también trajo trabajadores de Rosita como a Daniel Hernández, Pablo Pereda, Juan Pereda y Ernesto Olvera.

La historia a veces se ensaña y se repite de manera caprichosa; el taller del ferrocarril fue cerrado por decreto presidencial en atención a una necedad (así lo sostienen las viejas generaciones), La Consolidada, S.A. también fue cerrada por decreto presidencial en 1991 sin una plena justificación técnica y económica. Ambas empresas fueron el eje de la actividad económica de la ciudad por más de medio siglo; y se extinguieron por el presidencialismo; las dos se dedicaban a procesar acero, una a fabricarlo y la otra a darle forma; las dos fueron motivo de contratación de trabajadores y de servicios al resto de la ciudad.

Hasta principios de los años setenta, época en que se instala la industria maquiladora, se construye la planta Rassini Rem, empresa que produce autopartes (muelles y resortes), y la Compañía Carbonifera Río Escondido en 1974. La Consolidada, S.A. había sido la única fuerza impulsora del crecimiento económico, el aliciente de muchos pequeños negocios que dependían de los ingresos de sus trabajadores. Estos pequeños negocios vendían sólo algunos insumos a la empresa acerera. Cuenta Melchor 
Sánchez Jiménez ${ }^{3}$ que quienes promovieron la instalación de La Consolidada, S.A. fueron: Julio Santoscoy ("El viejo"), Arturo Flores Salinas, Ramón Purón, José de Luna, Generoso Montemayor y Salomón Libsón (Sánchez, 1990). ${ }^{4}$ Una generación de hombres con esta capacidad de gestión que logro la instalación de La Consolidada, S.A. en esta ciudad. ${ }^{5}$

\section{Primera etapa (1938-1957)}

Concluidos los trabajos de las instalaciones tanto del edificio como las bases de máquinas, la primera colada se hizo el 28 de mayo de 1938, fecha por demás arrogante que conjugo las temperaturas del clima de la ciudad de Piedras Negras, Coah. con el calor producido por el primer horno de hogar abierto con que inició la producción de acero La Consolidada, S.A. en esta ciudad fronteriza. Recuerda don Roberto Reyes que la primera colada consistio en $\mathbf{4 0}$ toneladas y dice que el personal:

[...] estaba distribuido así: en el horno de fundir, el fundidor y 2 ayudantes, en las tinas, un tinero que era el encargado de vaciar la tina llena de acero en los moldes y era el "mandamás" de ese departamento, un albañil con un ayudante para reparar tinas y canales. Dos graseros

3 Maestro de eđucación primaria y por muchos años presidente de la Sociedad de Maestros Coahuilenses. Fue también presidente municipal del municipio de Ocampo del estado de Coahuila, su tierra natal en los años setenta y ocupó otros puestos en el gobierno federal en el sexenio de Luis Echeverría. Su interés por la historia lo llevó a escribir un libro para el 140 aniversario de Piedras Negras, festejado en 1990.

4 Julio Santoscoy Perea: Fue agente del Ministerio Público en la Procuraduría General de la República de 1933 a 1941 . Ocupó el puesto de subsecretario del Trabajo y Previsión Social en el sexenio de López Mateos y ratificado en este mismo puesto por Gustavo Díaz Ordaz. Contribuyó al desarrollo de la ciudad de Piedras Negras; Arturo Flores Salinas: Originario de Zacatecas, fue propietario de la Compañía Nacional de Gas, que fundó en sociedad con Salomón Libsón, Max Lapin y los hermanos Montemayor, en 1934. La creación de esta compafía fue uno de los atractivos para instalar La Consolidada, S.A., en esta ciudad. Arturo Flores Salinas tuvo nexos muy estrechos con esta empresa que logró la concesión de una agencia aduanal, con que tramitaba las importaciones de chatarra y muchas de las materias primas e insumos para todas sus sucursales en el resto del país; Ramón Purón: Fue cofundador de los almacenes Trueba-Pardo. Junto con los hermanos Montemayor, Arturo Flores y otros empresarios formó la Compañ́a Nacional de Gas. La unidad de estos empresarios hicieron posible la creación de La Consolidada, S.A.; José de Luna: Fundador de la Cámara Nacional de Comercio en Piedras Negras en 1920. Fue también fundador del Casino Nacional y socio de la Compañía Nacional de Gas; Generoso Montemayor. Laboró en los Almacenes Trueba y Pardo, con el paso de los años la familia Purón se quedó con esta empresa; de igual forma, Refugio Montemayor logró hacerse socio de esta comercializadora y de la Compañía Nacional de Gas; Salomón Libsón: Hasta antes de crear la Compañía Nacional de Gas, tenía un negocio de frutas; este negocio le dio las ganancias para crear la compañía de gas. Se asoció también con Arturo Flores Salinas para crear dicha companíia. Actualmente sus hijos y nietos son los principales accionistas de esta compañía.

5 El sefior Antonio Reyes de la Riva, presidente municipal entre 1937 y 1938 , hizo las gestiones necesarias que condujeron al municipio a hacer la donación de los terrenos para instalar esta empresa. 
para tirar la grasa del acero, y 6 lingoteros que sacaban los moldes y los volvían a colocar para la siguiente carga. En el otro lado, por donde se cargaba el horno, un mayordomo con 6 hombres y un pesador, para cargar las cajas de fierro con chatarra para el horno (Reyes, 1996).

Mientras estos hombres enfrentaban el reto de producir acero en una pequeña ciudad como Piedras Negras prácticamente con nulas posibilidades de producir con él productos terminados, en México se constituía lo que hoy conocemos como la reforma agraria cardenista, que distribuyó la tierra a los campesinos. Este proyecto siderúrgico encabezado por una empresa estadounidense y la reforma agraria, habrían de coincidir en la necesidad de acero para labrar las tierras. Sin buenos tractores y arados con aceros resistentes a la fricción, difícilmente podía bacerse producir el campo mexicano. Aunque La Consolidada, S.A. tuvo por muchos años a los ferrocarriles y a las empresas mineras como su principal cliente y proveedor de chatarra, ya en estos años producía una diversidad de aceros en su planta La Ronda de la Ciudad de México.

Lázaro Cárdenas siempre aprobó la necesidad de hacer crecer la industria siderúrgica - a diferencia de Obregón y Calles-, incluso desde que fue gobernador de Michoacán (1928) luchó para que se explotaran las reservas de mineral de hierro Las Truchas, localizado precisamente en esa entidad. Tanto fue su empeño de que se incrementara la industria acerera nacional, y particularmente la explotación de estas reservas, que la empresa siderúrgica construida en 1975 en esta región recibió su nombre: Siderúrgica Cárdenas Las Truchas, S. A. (Sicartsa), y fue su primer presidente del consejo de administración.

En un contex to político favorable y un mercado totalmente virgen en constante expansión, más allá de la capacidad productiva de las empresas nacionales, la empresa fundidora de lingotes de acero, La Consolidada, S.A. sucursal Piedras Negras, fue bienvenida. A nivel nacional en esos años se colocaba como la principal productora de tuercas y tornillos, cuya cobertura en el mercado nacional era de $80 \%$; así como $100 \%$ en piezas de fundición para repuestos de la industria minera, $100 \%$ en las chumaceras para Ferrocarriles Nacionales y hasta 1946, cubria $80 \%$ del mercado de alambre de acero, fecha en que surge su competidor, Aceros Nacionales, fundado por Pablo Doitz, años atrás distribuidor de La Consolidada, S.A. (Zapata y Toledo, 1987).

Las condiciones propiciadas por la segunda guerra mundial, en las que su principal demanda y símbolo de poder de las potencias en lucha, era el acero, éste se presenta como el producto sin el cual no podía ganarse la batalla, ni desarrollar la industria de guerra, ni la manufacturera en general. 
Al inicio de la década de 1940 la producción de acero por todas las empresas mexicanas grandes y pequeñas, encabezadas por Fundidora Monterrey, S.A. y La Consolidada, S.A., según datos estimados, era aproximadamente de 130000 toneladas anuales, mientras que el consumo aparente era de 380000 toneladas; por lo tanto, las importaciones totales eran de alrededor de 250000 toneladas (Pape, 1949). Con ese mercado tan prometedor que se hacia cada vez mayor con el cierre de nuestras fronteras a las importaciones, propició que se fortaleciera esta empresa acerera.

Cuenta Roberto Reyes que: "En el año 1942 ya E.E.U.U. estaba en guerra. Pidió acero a La Concha. No recuerdo; pero creo fueron como 200 toneladas de acero de $4.5 \%$ de nickel, que es muy duro. Los hicieron rifles y cañones antiaéreos", según supo por conducto de uno de los altos funcionarios de apellido Seiffet" (Reyes, 1996).

La capacidad de producción de acero acumulada por la empresa a principios de 1940 era de 15000 toneladas de lingotes de acero mensuales (Juaristi y Jiménez, El Zócalo, abril de 1991). Todo este material era transportado por ferrocarril a la planta de laminación de La Ronda en el Distrito Federal. Como la demanda de productos de acero superaba la capacidad productiva de las plantas, La Consolidada, S.A. construyó una planta de laminación en Lechería, Estado de México en 1947; en aquellos años nuestra industria manufacturera aceleraba su ritmo de crecimiento, que se traducía en más pedidos de acero tanto para ampliar las instalaciones como para elaborar productos terminados.

En esta época, La Consolidada, S.A. se había convertido prácticamente en una empresa integrada por tres empresas productoras y varias bodegas distribuidoras en Guaymas, Sonora, Chihuahua, Chihuahua, Monterrey, Nuevo León, Guadalajara, Jalisco y Veracruz, Veracruz, situación que la hacía muy competitiva, con capacidad para soportar el fuerte auge que venían adquiriendo Altos Hornos de México, S.A. (empresa paraestatal recientemente fundada en 1944), Hojalata y Lámina, S. A. y Fundidora Monterrey, S.A., esta última contemporánea suya de principios de siglo.

Además, las fuertes demandas de lingotes de acero de diferente composición química y térmica demandadas por el mercado, propiciaron la instalación del segundo homo de aceración Siemens-Martin en la planta de Piedras Negras en 1947. Este segundo horno aumentó la capacidad productiva de la planta precisamente para abastecer también a la planta de laminación de Lechería, Estado de México, que se dedicaría a producir

6 Siemens-Martin: horno de aceración conocido como de hogar abierto. Funciona también por medio de soplo y el acero se obtiene agregando chatana y aleaciones de metales, dependiendo del tipo de acero que se trate. 
perfiles, con una capacidad de hasta 10000 toneladas mensuales. En 1956 se instal6 el tercer horno de aceración, y en 1957 logró su integración total al instalar su primer alto homo ${ }^{7}$ para producir hierro de primera fusión.

Este primer alto homo había sido abandonado en condiciones de chatarra en Rusk, Texas; fue adquirido por esta empresa como producto del agotamiento de la chatarra en el sur de Estados Unidos. Ya desde antes que se presentara la necesidad de producir acero a través de arrabio, ${ }^{8} \mathrm{La}$ Consolidada, S.A. había adquirido lo que hoy conocemos como La Perla, S.A., mineral de hierro localizado en el estado de Chihuahua en las fronteras con Coahuila. Harry Wright comisiono a un ingeniero de apellido Hudson para localizar minas en el estado de Chihuahua; encontró un mineral de hierro a nombre de un francés llamado Pablo Gunter que desde 1888 había adquirido los derechos, pero por falta de recursos nunca pudo explotarla (Zapata y Toledo, 1987). Producto de esta situación de ociosidad e indefinicion de este mineral, Wright la denunció a nombre de $\mathrm{La}$ Consolidada, S.A. A 20 kilómetros de La Perla fue encontrado otro yacimiento denominado La Negra, que pronto se agotó.

Como un dato controvertido hay que decir que en 1942 La Consolidada, S.A. vendió La Perla a un grupo de accionistas de Wall Street encabezado por Paul Shields, quien posteriormente creó el consorcio Minas del Norte. La Consolidada, S.A. continuó sus nexos con el sector minero a través de Víctor N. Agather, socio y vicepresidente del consejo de administración, que por muchos años fue también minero. Aun cuando vendio sus minas puđo abastecer de hierro del subsuelo a su primer alto horno, que inició con una capacidad de 200 toneladas diarias de producción de arrabio. Este horno, uno de los más pequeños del mundo, fue ampliado en su capacidad de producción hasta 350 toneladas diarias. Ese mismo año Altos Hornos de México, S.A. adquirió La Perla bajo presión de Harold Pape sobre Víctor N. Agather, que siempre había deseado anexar al citado consorcio (Zapata y Toledo, 1987).

Con la instalación del alto homo en 1957 concluye una etapa de prosperidad para La Consolidada, S.A. Precisamente cuando inicia su producción de acero con la combinación AH-HA (alto homo-hogar abierto todavía la principal tecnología para producir acero en el mundo), se ve obligada a deshacerse de sus minas de hierro. Por fortuna la magnitud de

7 Horno de cuba profunda, vertical, provisto de soplo para producir arrabio por reducción de minerales de hierro, carbón coque, caliza y dolomita; tiene la forma de dos troncos de cono unidos por su base mayor. Se construye de acero y su interior es revestido de ladrillo refractario.

8 Mezcla de fierro, carbón coque, dolomita y caliza fundidos a más de 1500 grados centígrados en el interior de los altos hornos para la fabricación de acero. 
su alto horno no hacía necesario la propiedad de tan grandes reservas de mineral de hierro como fue La Perla, que Altos Homos de México, S.A. sí necesitaba porque ya para entonces había instalado su segundo alto horno en septiembre de 1954, con una capacidad de 1000 toneladas de arrabio diarias. En 1957, la pequeña fundidora de acero de Piedras Negras había adquirido la capacidad representada en el siguiente cuadro:

\section{CUADRO 1. Piedras Negras, Coahuila, La Consolidada, S.A. Capacidad y fecha de instalación de los hornos de hogar abierto y el alto horno.}

\begin{tabular}{cc}
\hline Fecha & Toneladas por colada \\
\hline 1938 & 80 \\
1947 & 100 \\
1956 & 100 \\
Alto homo, 1957 & 350 al día \\
\hline $\begin{array}{c}\text { Fuente: Información proporcionada por el Ing. Magdaleno Zavala Morales, último } \\
\text { gerente de la empresa. }\end{array}$
\end{tabular}

Segunda etapa (1958-1982)

La Consolidada, S.A, empezó a tener problemas financieros en el momento en que se vio obligada a vender sus minas de Chihuahua. Zapata y Toledo afirman que su programa de inversiones para mejorar sus plantas y reducir costos se habían hecho dentro de un límite razonable, no obstante, $i$ cómo se explica que en 1958 cambia de dueño? Mike Feldman, un ruso-alemán nacionalizado mexicano, y que había sido distribuidor en el Distrito Federal, compra la empresa. En sus manos inicia el desmantelamiento; la empresa Condumex compra el departamento de Conductores Eléctricos; en 1966 trasladan la mayor parte de los departamentos de La Ronda a San Martín en el Estado de México, muy cerca de la planta de Lechería.

Sólo cuatro años estuvo en manos de Mike Feldman esta gran empresa acerera. En el año de 1962 pasa a formar parte del consorcio AHMSA. Las razones: problemas para continuar produciendo con márgenes de rentabilidad aceptables. La planta de Lechería, Estado de México, la de Piedras Negras junto con sus bodegas en distintas partes del país, pasaron a propiedad del gobierno federal y a consolidar una estrategia de crecimiento económico con un apoyo decidido por parte del gobierno, a través de acero 
financiado con gasto público, hecho que fortaleció la participación del Estado en la economía.

El gobierno se convirtió a partir de esos años en el principal productor y distribuidor de acero, cuyo destino fue apoyar el proceso de industrialización por sustitución de importaciones; una estrategia de crecimiento industrial distinta, cuyo eje central era proteger a la industria nacional naciente, de las importaciones, lo que pedía La Consolidada, S.A. en los años veinte cuando gobernaron México el general Álvaro Obregón y Plutarco Elías Calles, cuyo apoyo fue dirigido más al sector minero y a las empresas importadoras.

De 1958 a 1962 es un periodo de cinco años muy corto para una empresa siderúrgica que había logrado recientemente su integración total, y dada la situación de crecimiento de la economía nacional, tenía un futuro asegurado para la venta de sus productos. No obstante, seguramente por la fuerte competencia que ejercían las dos recientes grandes empresas siderúrgicas instaladas en el año de 1944; Hojalata y Lámina. S.A. y Altos Hornos, de México, S.A., propició la contracción de sus ventas; antes de que esto sucediera, Mike Feldman obtuvo todo el provecho posible (según aseguraban todos los entrevistados que trabajaron en la empresa durante esos años).

Quizás estemos equivocados con la periodización de la historia para esta empresa, particularmente en la segunda etapa que inicia realmente a partir de 1962, cuando la pequeña planta de la ciudad pas6 a pertenecer al gobiemo. Desde antes que se llevara a cabo la transacción de La Consolidada, AHMSA tenía acciones de ella; en esa situación no fue muy difícil para el gobiemo adquirirla, hecho que hizo a través de un trueque de acciones intercambiando cinco acciones de La Consolidada,S.A. con un valor nominal de 75 pesos cada una, por tres de AHMSA pero con un valor nominal de cien pesos cada una (Zapata y Toledo, 1987). Con la venta de esta acería al gobiemo, se cierra poco más de medio siglo de vida de una empresa que jugó un papel importante en la producción de acero de calidad para la fabricación de refacciones, herramientas y accesorios para nuestra industria manufacturera.

Pasar a propiedad del gobierno en una época de crecimiento económico por arriba de $6 \%$ como promedio anual, justo en el momento cuando inicia lo que llamamos "El periodo estabilizador" de la economía nacional, cuyos indicadores básicos fueron tasas inflacionarias de $3 \%$ y un tipo de cambio que puso el dólar a $\$ 12.50$, parecería poco creíble, pero así ocurrió. Con estos acontecimientos, se materializa lo que alguna vez Harry Wrigth le dijo a Obregón sobre la importancia de tener una industria siderúrgica fuerte (Zapata y Toledo, 1987), En particular, Altos Homos de México, 
S.A. en esos años se acercaba a la producción del millón de toneladas de acero líquido, cifra que logró en 1964 con la instalación de su octavo homo de aceración Siemens-Martin en marzo del año en cuestión. Más los tres hornos de aceración de Piedras Negras, Coahuila que producían en promedio 180000 toneladas de acero en lingotes, pasó a convertirse en la principal empresa siderúrgica en América Latina.

Por muchos años La Consolidada, S.A. produjo aceros de distintos calibres y para distintos usos, incluyendo el acero antifricción (babbitt) para las chumaceras de los ferrocarriles. Cuando pasa a propiedad del gobierno, el tipo de acero cambió de calidad y la variedad de su composición química; de igual forma los movimientos comerciales para su proceso de laminación y venta. A partir de 1962 los lingotes de acero que producía La Consolidada, ahora Altos Hornos de México, S.A. planta Piedras Negras, (AHMSA, Piedras Negras) empezaron a llevárselos a Monclova, Coahuila, a sus plantas de laminación para la producción de aceros planos.

Al pasar a propiedad del gobierno, esta pequeña acería fue relegada y se colocó en una situación de desventaja para darle un buen mantenimiento a sus principales departamentos. No obstante, la pequeña acería continu6 produciendo a pesar de que las fuentes de suministro de materias primas (hierro y chatarra) cada vez se hacían más caras, tanto por el costo del uransporte como por la escasez de esta última. En un periodo como en el que le tocó pertenecer al gobiemo, conocido como "Milagro mexicano" por la bonanza de su economía, aun cuando se le relegaba, el mercado demandaba mucho acero, fortaleciendo su permanencia en aquél. Esta situación cambió bruscamente a principios de la década pasada. El consumo aparente inició un proceso de contracción de cerca de $50 \%$ del consumo nacional en 1983, lo cual significó un duro golpe al sector a nivel nacional y la pequeña planta de Piedras Negras no pudo salvarse de sus efectos devastadores.

Desde que instaló su alto horno en 1957 y hasta 1983, no experimentó cambios importantes en sus departamentos: mantuvo su equipo funcionando sin innovaciones tecnológicas, e incluso, con poco presupuesto para darle mantenimiento a su maquinaria. Por esta situación, los costos de producción con los homos de aceración Siemens-Martin se incrementaron muy por arriba en comparación con el nuevo taller de aceración al oxígeno (BOF) instalado en la siderúrgica de Monclova, Coahuila en 1971. Hacia 1983 esta tecnología era obsoleta dentro de AHMSA Piedras Negras. En ese contexto se inició el proceso de reestructuración de la pequeña planta, del cual hay que destacar el fuerte conflicto de intereses y una férrea oposición a cerrar los homos de aceración; decisión que fue concretada en 1983. 


\section{Tercera etapa (1982-1991)}

La crisis económica que inició en 1982 con una caída en el producto interno bruto (PIB) particularmente brusca, repercutio en todos los sectores y ramas industriales; de haber registrado una tasa de crecimiento arriba de $8 \%$ durante 1981, en 1982 fue cero porciento. Los recursos del petróleo se agotaron al contraerse el mercado, más la deuda acumulada con la banca extranjera que rebasaba los 80000000000 de dólares, se tradujeron en fuertes desequilibrios estructurales. Si en la época de bonanza petrolera los recursos que generaba este sector no alcanzaban para pagar los intereses de la deuda, al bajar el precio hasta 8 dólares por barril, el impacto recesivo fue determinante para caer en crisis.

La industria siderúrgica nacional, que tiene como uno de sus principales clientes a Petróleos Mexicanos, inmediatamente sintio el impacto de la falta de pedidos de tubería, lámina y hojalata para el enlatado de los aceites y lubricantes, así como para la conexión de los oleoductos y gasoductos. Con este cambio brusco en el mercado mundial del petróleo, más la acumulación de desequilibrios con el sector externo como producto de la inflación, así como el alto déficit gubernamental a que se llegó por financiar al sector paraestatal en constante quiebra, condujeron a la peor crisis que había conocido México desde 1929.

La industria siderúrgica mexicana "camuflada" por el proteccionismo estatal desde los años cuarenta, esta vez no pudo quedar al margen de la recesión. Si en 1975 se salvó de la severa crisis que el sector sufrió a nivel mundial y que obligó a los países líderes a reestructurarse cerrando empresas enteras (este tipo de industria es de lo más antiguo y tradicional que existe), en México sólo siete años duró el proteccionismo, que promediaba $40 \%$ del costo total en las importaciones de productos siderúrgicos.

Como producto de esta situación de quiebra total del sector, el gobiemo mexicano, propietario del porcentaje más elevado de la producción y distribución de productos siderúrgicos a través de Sidermex (paraestatal que se constituyó en 1978 para administrar a Fundidora Monterrey, S.A., Altos Hornos de México, S.A. y Sidenúrgica Lázaro Cárdenas Las Truchas, con todas sus empresas filiales), se vio en la necesidad de iniciar un proceso de reestructuración que comprendiera todo el sector, desde las cuestiones laborales referentes al contrato colectivo de trabajo, pasando por las formas de organizar y repartir las tareas entre los trabajadores, así como introducir mejor maquinaria y en algunos casos, nueva tecnología con procesadores digitales. Definitivamente se llegó también a la necesidad de cerrar a Fundidora Monterrey, S.A., pionera del sector desde principios de siglo, así como departamentos enteros como los de aceración Siemens-Martin 
en las plantas de Monclova, Coahuila que quedaron predestinados, para concluir su periodo de vida a principios de esta década.

Altos Homos de México, S.A. de Piedras Negras recibió su primera amenaza de cierre en esta época; el periódico de circulación regional, El zócalo, el 15 de febrero de 1983, hizo público en estos términos dicha amenaza de cierre con el siguiente título:

\section{"OFICIAL: CIERRA AHMSA"}

La empresa Altos Hornos de México hizo oficial ante el Sindicato de Trabajadores Mineros Metalúrgicos de la República Mexicana el cierre definitivo de la planta local y la terminación de las 720 gentes tanto sindicalizados como empleados de confianza que allí laboran. Arturo Ruiz Chavez, Director corporativo de AHMSAen comunicación enviada al Secretario General del Sindicato, Napoleón Gómez Sada, le dió a conocer la decisión del consejo de la empresa señalándole como puntos fundamentales del cierre la falta de mercado y el alto costo de la planta la cual además de no producir está resistiendo pérdidas mensuales del orden de los 10 millones de pesos (El zócalo, 1983).

En esta misma noticia se hacía referencia al golpe económico que representaba para Piedras Negras, Coahuila cerrar una industria y una fuente de trabajo, que cinco años después, concluiría medio siglo de generar ingresos para la ciudad y sus habitantes. Además, representaba un símbolo de identificación, algo que ninguna otra ciudad fronteriza poseía. La Consolidada, S.A. en su tiempo, y Altos Hornos de México, S.A. Piedras Negras, posteriormente, imprimieron un especial estilo de ser de los habitantes de esta ciudad; una cultura industrial que con su cierre, se veía amenazada.

La pequeña empresa acerera se salvó de los efectos de salir del mercado con la política de reestructuración industrial impulsada por el gobiemo federal; no obstante, se pensó en la posibilidad de cambiar de giro y en lugar de producir acero, convertirla en una fundidora en apoyo de todo el sector paraestatal. Además, brindar servicios de reparación y rectificación a los equipos del resto de las empresas del consorcio, en particular a las plantas de Monclova,Coahuila, las principales del consorcio AHMSA.

Precisamente en eso consistió la reestructuración de AHMSA, Piedras Negras a principios de la década pasada; esta estrategia del gobierno puso en aprietos no sólo a la pequeña acería, también a toda la economía de la ciudad de Piedras Negras, Coahuila que tenía una relación de dependencia muy fuerte con ella. La obsolescencia de su maquinaria, en particular del 
horno de aceración de hogar abierto, muy costoso y contaminante, la obligaron a cambiar de giro. En esos años ya, en Monclova, Coahuila se obtenía acero por medio de convertidores al oxígeno desde 1971, sistema que era más económico.

Para iniciar el nuevo proyecto de reestructuración productiva, se hizo necesario invertir 540000000 de pesos, repartidos de la siguiente manera: 150000000 para reparación del pequeño alto horno; 200000000 para hacer crecer el taller de fundición y 190000000 para el traslado e instalación del horno de cubilote desde Monterrey, Nuevo León, cuya capacidad era de 25 toneladas por hora (AHMSA-Avante, 1984).

A partir de 1984, lo que años atrás fue una empresa acerera de mucha importancia por la calidad de su acero, se dedicó a producir lingoteras, bases y ollas para AHMSA de Monclova, mediante la utilización de arenas furánicas para el moldeo. La nueva empresa reestructurada logró una capacidad de 5000 toneladas por mes de sus nuevos productos. Posteriormente, ya como parte del nuevo perfil, se invirtieron 300000000 de pesos para la instalación de un taller de recuperación de rodillos de laminación con una capacidad de 220 por año; todo este material era destinado a las plantas de Monclova. Con estas inversiones que remodelaron y cambiaron de giro a la acería de esta ciudad para hacerla rentable ante un mercado deprimido, se recuperó el empleo perdido un año antes y es así como se obtuvieron los primeros frutos del proceso de transformación del proceso de reestructuración (AHMSA-Avante, 1984).

Cuentan quienes estuvieron dirigiendo a AHMSA de Piedras Negras en esta época difícil, que se dio una recuperación impresionante en sus cuentas, contrario a lo que el 15 de febrero de 1983 se había publicado en la prensa, en donde se afirmaba una pérdida de 10000000 de pesos mensuales. Cuenta el Ing. Magdaleno Zavala Morales (último gerente de la empresa) que AHMSA de Monclova sólo utilizaba una vez los rodillos de laminación; posteriormente, y como producto de la crisis económica, fue necesario rectificarlos para usarse por lo menos tres veces en diferentes niveles de los molinos desbastadores. Cada rodillo costaba en aquella época entre 3000000 y 5000000 de pesos; cuando construyeron y adaptaron el taller de reparación para rectificación de rodillos en esta planta, el ahorro para Monclova, Coahuila, fue cuantioso; además, AHMSA de Monclova compraba las coquilas en Fundiciones Monclova, S.A. (fundidora privada que evidentemente representaba un mayor costo para el consorcio que si se hacían por cuenta propia en los talleres de Piedras Negras).

Con este nuevo giro industrial, a semejanza de una empresa maquiladora, AHMSA de Piedras Negras continuó trabajando hasta 1991. 
El 10 de mayo de ese año, El zócalo, periódico de circulación regional anunciaba con grandes encabezados: "Confirmado: cierra AHMSA". El motivo: el exceso de contaminación que generaba el homo de cubilote que deterioraba agua, tierra y aire; la Secretaría de Desarrollo Urbano y Ecología (Sedue) tomó la decisión de cerrar. Cuentan quienes les tocó vivir la experiencia, que Sedue, sin previo estudio que demostrara el nivel de contaminantes, cerro la empresa que por muchos años dio trabajo a los habitantes de esta ciudad. Su cierre definitivo representó un duro golpe, tan fuerte que todavía se reciente la pérdida de más de 700 empleos.

\section{La importancia económica a nivel regional}

Construir una planta fundidora para obtener productos de acero en una pequeña ciudad que había sufrido los efectos devastadores de la recesión de 1929 a 1933, y que además, por capricho del presidente Plutarco Elías Calles se llevaba la única fuente de empleo, La Maestranza, a otra ciudad del centro de México; de esta forma La Consolidada, S.A. se convirtió en una bendición para Piedras Negras que vino a suplir a los talleres del ferrocarril creando empleos e ingresos.

En sus Recuerdos de La Concha, comenta don Roberto Reyes (1996), que en el treinta y seis no había camiones urbanos como ahora, y para transportarse a la colonia Morelos, lugar donde se estaban construyendo sus instalaciones, lo hacían en bicicletas. El uso de la bicicleta motivó la creación de pequeños talleres para darles mantenimiento. Es así como se empezaron a sentir los efectos de inducción de la planta acerera sobre la atrofiada economía de la ciudad.

Tan poca era la capacidad para abastecer de insumos a la pequeña planta, que la madera que se usó para los trabajos de albañilería y toda la construcción que necesitó este material, fue importada de Eagle Pass, de la maderería Estrada Lumber, así como el gas natural, que proporciono don Arturo Flores. Para abastecer de este insumo básico a la planta fue necesario atravesar con tubería el río Bravo. Estas construcciones y el consumo de gas de la vecina ciudad estadounidense propiciaron derramas económicas importantes para su época y el nivel de desarrollo de aquellos años en la comunidad.

Al finalizar la década de 1930, el mundo entró en guerra y los Estados Unidos cambiaron el giro de su industria para producir pertrechos de guerra, lo que impiđió por algunos años la producción y comercialización de autos; sólo hasta después de 1945 empezaron a entrar a esta ciudad. Dice don Roberto Reyes (1996) que esto motivó el surgimiento de talleres de mecánica para arreglar carros, al mismo tiempo que se inició el 
comercio de partes automotrices estadounidenses. Hay que decir que los ingresos que generaba la empresa hacían posible comprarlos con muchas facilidades en la ciudad vecina.

Con la construcción de la planta de cal en 1954, la acería incremento sus pedidos de piedra caliza, materia prima que se obtenía en las riberas de los ríos Bravo y Escondido; este negocio duró hasta el año en que $\mathrm{La}$ Consolidada, S.A. pasó a propiedad de la paraestatal Altos Homos de México, S.A. Cuenta Roberto Reyes (1996) que se consumían cerca de 30 toneladas diarias, cuyo uso servía para reducir los concentrados de azufre, mineral que hace difícil el proceso de laminación. Uno de los personajes que se beneficio con el suministro de esta materia prima fue Carlos Mondragón. En resumen, el tipo de materia prima e insumos que la empresa compraba al comercio de la localidad fueron; piedra caliza hasta principios de los años cincuenta; herramientas pequeñas de mano que compraban a refaccionarias como Casa Villarreal; en su última etapa se compró leña para el cubilote y toda la papelería y artículos de oficina se adquirían en la ciudad, en la papelería Bravo.

Cuando pasa a formar parte de AHMSA, mucho de lo que compraba a los negocios de Piedras Negras, Coahuila e Eagle Pass, llegaban desde Monclova, Coahuila. El resto de la materia prima, como la chatarra, era importada de Estados Unidos; el mineral de hierro, de La Perla; el Coque, en sus tiempos de empresa privada, se lo compraba a Asarco; los metales para obtener las aleaciones, de Monclova y otras ciudades del país. Como puede observarse, había muy poca integración hacia atrás y hacia adelante, ninguna; todo el lingote de acero se procesaba en otras ciudades del país, cerca de los mercados de consumo.

Los hombres que más se beneficiaron con la instalación de La Consolidada, S.A. en Piedras Negras, fueron sus promotores. Los Almacenes Montemayor, Trueba Elosua y Casa Villarreal proporcionaban la mayoría de los insumos y servicios que adquiría en esta ciudad. Estos negocios pertenecieron a Refugio Montemayor; Ramón Purón y Lucas Villarreal, respectivamente. No sabemos si la venta de estos servicios e insumos a la empresa acerera jugo un papel importante en el creciminto de sus negocios; no obstante, por medio de esta relación se generó algún "escurrimiento" financiero a favor de empresas y empresarios locales. Si la empresa en cuestión hubiese decidido obtener mayor integración con los negocios de la ciudad, se habría capitalizado más.

Seguramente la poca capacidad de las empresas de la ciudad para abastecerla de sus principales insumos, obligó a la acería a comprarlos fuera de la ciudad. Esta situación empeoró aún más cuando paso a formar parte del consorcio AHMSA. Prácticamente la mayor parte de las materias 
primas e insumos se traían de Monclova, Coahuila. Si nos viéramos en la necesidad de confirmar esta relación comercial con la planta acerera, seguramente encontraremos este cambio brusco a favor de la economía de Monclova.

Al pasar a formar parte de AHMSA, La Consolidada, S.A. de Piedras Negras, se colocó en una situación de total dependencia que no había la posibilidad de continuar con sus propios mercados; así lo confirman todos los entrevistados que alguna vez dirigieron esta empresa. Su capacidad muy modesta, así como lo obsoleto de sus principales máquinas, hacían difícil que se moviera con recursos propios para encontrar nuevos nichos de mercado; en 1991, año en que fue cerrada, producía sólo $6.8 \%$ del total de AHMSA.

Hay consenso en que su mayor impacto económico fue por medio de la derrama salarial. Según confirma Javier de Luna, contralor de AHMSA Piedras Negras por muchos años, que $80 \%$ de los ingresos que generaba era por medio de salarios. Con su cierre, los comerciantes de la ciudad se solidarizaron con los obreros despedidos al ver que una de sus fuentes de ingreso desapareció. El viernes 19 de abril de 1991, Francisco Juaristi Santos, propietario de El zócalo, en la sección local del diario publicó cifras proporcionadas por directivos de la empresa en los siguientes términos:

$$
\text { "180 MUls., salarios en AHMSA" }
$$

Ciento ochenta millones de pesos es la derrama económica semanal de Altos Hornos de Méxíco, entre trabajadores sindicalizados y empleados de confianza [...] Los 423 trabajadores de planta reciben cada semana un total de 106 millones de pesos, más 5 millones en bonos de despensa. Este último es de 12,500 por persona. Los empleados de confianza obtienen, en el mismo lapso, 74 millones ( $E l$ zocalo, abril de 1991).

El pueblo de Piedras Negras, Coahuila en su conjunto no estuvo de acuerdo con este hecho; correspondía al mercado cerrarla por incosteable, como estuvo a punto de ocurrir en el año de 1982 cuando se contrajo el mercado del acero. Al igual que en aquel año, existía la esperanza de que se abriera porque había sido una decisión de una autoridad ajena a la organización y además, no había demostrado los niveles de contaminación que generaba la empresa acerera.

La principal "correa de transmisión" del crecimiento económico hacía el resto de la ciudad fueron los salarios de sus trabajadores. Desde 1936, año en que iniciaron su construcción, empezó a pagar 1.50 pesos diarios; posteriormente, sus salarios superaron al resto de las empresas de la 
localidad (cuadro 2). Aunque el privilegio de ganar más en salario nominal en comparación con Rassini, Micare y algunas empresas maquiladoras, se perdió en sus últimos años de existencia, afirma Héctor Efraín Sonora, que en términos de prestaciones siempre tuvo las más altas de la ciudad.

Un aprendiz de fundidor ganaba en 1967,1.23 pesos más que el salario mínimo vigente; en cambio, el fundidor ganaba arriba de 3.7 salarios mínimos; a esto hay que agregar el porcentaje de prestaciones que superaba a otros negocios de la ciudad. Es cierto que el salario mínimo sólo es un indicador de referencia para el pago de salarios por las empresas y que por lo común pagan más; no obstante, en aquellos años, la mayor parte de las organizaciones de esta ciudad eran microempresas que no podían pagar mejores salarios que la única gran empresa de la ciudad. Al instalarse la maquila y otras de mayor tamaño, la competencia por la mano de obra se incrementó notablemente, aún así los acereros de esta ciudad se mantuvieron en una posición de ventaja.

\section{CUADRO 2. Piedras Negras, Coahuila. Altos Hornos de México,} S.A. Comparativo salarial 1967-1989.

\begin{tabular}{|c|c|c|c|c|c|c|c|c|}
\hline Años & $\begin{array}{l}\text { Salario } \\
\text { mínimo } \\
\text { general }\end{array}$ & $\begin{array}{c}\text { Aprendiz } \\
\text { horno } \\
\text { open }\end{array}$ & Cargador & $\begin{array}{l}\text { Ayudante } \\
\text { de tinero }\end{array}$ & $\begin{array}{c}\text { Ayudante } \\
\text { de } \\
\text { mecánico }\end{array}$ & $\begin{array}{l}\text { Ayudante } \\
\text { operador } \\
\text { de grúa }\end{array}$ & Velador & Fundidor \\
\hline 1967 & 22.15 & 27.29 & 37.64 & 38.93 & 39.47 & 41.70 & 41.58 & 81.67 \\
\hline 1969 & 26.00 & 31.25 & 43.10 & 44.57 & 45.19 & 47.75 & 47.61 & 93.49 \\
\hline 1971 & 29.80 & 35.94 & 49.56 & 58.73 & 51.97 & 54.91 & 54.75 & 107.51 \\
\hline 1973 & 41.55 & 41.33 & 56.99 & 57.86 & 59.77 & 63.15 & 62.96 & 123.64 \\
\hline 1977 & 95.20 & 108.28 & 149.30 & 154.42 & 156.55 & 165.43 & 164.93 & 323.88 \\
\hline 1979 & 122.00 & 149.57 & 196.13 & 201.94 & 204.36 & 214.44 & 248.16 & 394.28 \\
\hline 1981 & 170.00 & 305.26 & 305.26 & 314.30 & 318.06 & 363.06 & 386.23 & 613.66 \\
\hline 1983 & 421.00 & 659.73 & 687.39 & 838.24 & 683.39 & 870.15 & 719.39 & $1,216.8$ \\
\hline 1985 & 1,015 & $1,532.1$ & $1,857.26$ & $1,727.50$ & $1,585.44$ & $1,532.10$ & $1,647.16$ & $2,606.5$ \\
\hline 1987 & 3,7504 & $4,651.47$ & $4,755.80$ & $5,442.92$ & $4,774.08$ & $5,584,39$ & $5,427.35$ & $7,121.5$ \\
\hline 1989 & 7,6401 & $14,077.4$ & $15,061.8$ & $14,393.2$ & $14,448.5$ & $14,077.4$ & $14,877.9$ & 21,555 \\
\hline
\end{tabular}

Fuente: Contrato Colectivo de Trabajo AHMSA, Altos Homos de México, S.A. Planta Piedras Negras, 8 de marzo 1979. Gobierno del estado de Coahuila, Junta Local de Conciliación y Arbitraje.

\footnotetext{
9 Penúltimo secretario general de la sección 123 del Sindicato Nacional de Trabajadores Mineros, Metalúrgicos y Similares de la República Mexicana (SNTMMSRM).
} 
Todos estos privilegios no sólo hay que atribuirlos a la capacidad y solvencia de la empresa para pagar buenos salarios a sus trabajadores; también el sindicato minero jugó un papel decisivo. La tradicional lucha de los mineros de esta parte de México, que se convirtió con el paso de los años en una fuerza de negociación, contribuyó para que estos trabajadores llegaran a ser una especie de élite dentro de la clase obrera de Piedras Negras, Coahuila.

La sección 123 del Sindicato Nacional de Trabajadores Mineros, Metalúrgicos y Similares de la República Mexicana (SNTMMSRM), hizo también contribuciones importantes al desarrollo de la comunidad: logro la suficiente capacidad financiera como para construir el estadio que lleva su nombre; asimismo, hizo donaciones para la construcción de dos escuelas primarias. Todo esto pasó a formar parte de la historia de Piedras Negras que necesitamos reconstruir. Sus enseñanzas y sus recuerdos servirán de testimonio para hacerle frente a un mejor futuro.

\section{CONCLUSIONES}

La herencia económica y cultural que dejo La Consolidada, S.A. en el pueblo de Piedras Negras, Coahuila es un patrimonio con una magnitud poco valorada, incluso por las generaciones que les toc 6 trabajar, construir y mantener por más de medio siglo a esta empresa. Son pocos los hombres que están dispuestos a proporcionar sus conocimientos y recuerdos para dejar por escrito toda su historia y su importancia económica en el contexto de la ciudad y la región norte de Coahuila. Con esos pocos y la escasa información de archivos, haremos el intento de escribirla para dejar testimonio de aquellos hombres que enfrentaron el calor de los hornos para producir acero.

El presente escrito es sólo un primer intento; es un bosquejo de sus principales etapas, del tipo de acero que producía y las máquinas que adquiri6. De igual modo, como ha podido observarse, es un intento de interpretar su relación con la economía de la ciudad. Al respecto, es claro lo poco significativa de su integración hacia atrás comparativamente con el costo total de las materias primas e insumos que necesitaba, tanto en su etapa inicial como en sus últimos años de existencia.

La escasa integración hacia atrás y la nula integración hacia adelante tiene su explicación en la pequeñez de los negocios de esta ciudad. Sus empresarios no tuvieron la capacidad de traer toda la materia prima, pero sobre todo, algunos de los insumos de tamaño menor cuya demanda cotidiana les permitiera crecer y acumular algún capital de la magnitud generada en Monterrey, Nuevo León con las empresas acereras, o como 
en Monclova, Coahuila, como lo fue Refractarios Básicos, S. A., empresa que abastece de cal siderúrgica a AHMS A de esa ciudad. Evidentemente que La Consolidada, S.A. fue una empresa más modesta, pero también muy integrada que se proveía de la mayor parte de sus insumos sin necesidad de intermediarios; además, mucha de su materia prima era difícil de obtener en la región, como la chatarra e incluso, el mineral de hierro.

Su integración con la economía de la ciudad se llevó a cabo por medio de los ingresos de sus trabajadores. El comercio organizado capitalizó dichos ingresos; no obstante, los trabajadores pudieron mantener a sus familias, construir sus casas y darle educación a sus hijos. Cuando esto se acabó, representó un duro golpe para la economía familiar y para el comercio organizado. Después de cinco años de haber cerrado, parece que las heridas están cicatrizando. Un alto porcentaje de sus trabajadores están ya ocupados en otras labores, pero la empresa y la experiencia enviada al vacío, jamás se recuperan.

\section{BIBLIOGRAFIA}

AHMSA-Avante, revista de publicación interna, varios números.

PAPE, Harold R. "Los primeros seis años de Altos Hornos de México". Conferencia impartida en la Universidad de Texas en Austin, el 11 de junio de 1949.

ZAPATA, Francisco y Daniel Toledo. 1987. La Consolidada: de fundición a empresa integrada. Inédito; México, El Colegio de México, Centro de Estudios Sociológicos.

REYES, Roberto. 1996. "Recuerdos de La Concha". Inédito, Piedras Negras, Coahuila.

Sindicato de Trabajadores Mineros y Metalúrgicos de la República Mexicana, sección 123, Contratos Colectivos de Trabajo, 1967-1989.

JMÉNEZ Sánchez, Melchor. 1990. Breve historia de Piedras Negras. Conmemoración del 140 aniversario, Ayuntamiento.

El zócalo, periódico de circulación regional, varios números. 